\title{
Past, present, and future in pediatric spinal surgery
}

\author{
Jean Dubousset
}

Académie Nationale de Médecine, rue Bonaparte, Paris, France

Correspondence to: Jean Dubousset. Académie Nationale de Médecine, 16 rue Bonaparte, 75006 Paris, France. Email: Jean.dubousset@wanadoo.fr.

\begin{abstract}
According the almost 55 years of experience in Pediatric Spinal surgery it was easy for me to describe the evolution during the past of the surgical techniques as well as the indications for spinal deformities done first without any instrumentation (still useful from time to time nowadays), as from the back as on the front, post-operative immobilization achieved thanks to casting. The real instrumentation appeared successively with Harrington, Luque, and simultaneously the introduction of pedicle screw thanks Raymond Roy Camille. It was necessary to wait another 20 years to get the segmental 3D strategy of the CD instrumentation still the basis of modern spinal surgeries techniques whatever using Hooks Screws, Universal clamps or Hybrid constructs. For present \& future, Early surgery is still indicated for localized lesion generally secondary to congenital malformations with or without spinal cord decompression. But for extended lesions especially involving the thoracic area cast and brace is still the good choice whatever the etiology with or without pelvic obliquity. When this treatment fails many attempts with various techniques were used with some success for spinal growth without disturbances about the respiratory function. But it was also demonstrated that the number of complications were still high, with in many cases the necessity to perform a final surgical fusion. It is why the development of the bipolar minimal invasive technique appeared, with very promising results, including the fact that a substantial number of patients get a spontaneous fusion, excluding final surgery. For the older or adolescent patients, the evolution toward a race to obtain the maximum correction of the Cobb angle become more and more disputable as is was demonstrated that the most important for the future regarding the spinal function is the $3 \mathrm{D}$ dynamic balance of the discs spaces left free below and above the fused area.
\end{abstract}

Keywords: 3D; bipolar minimally invasive surgery; pelvic obliquity; 3D balance

Submitted Jul 03, 2019. Accepted for publication Jul 24, 2019.

doi: $10.21037 /$ atm.2019.08.13

View this article at: http://dx.doi.org/10.21037/atm.2019.08.13

\section{Introduction}

The development of spinal surgery at the pediatric age followed in fact the one of general spinal surgery thanks mainly the improvement of the anesthesiology techniques as well as the post-operative care. Initially, the spinal deformities in children and adolescents were treated not operatively from a long time by external corrections tractions cast and braces of various nature. But it appears only slowly that the surgical approach of the spine done sub-periosteal, gave, either in the back or even in the front, an early fusion of the approached area, with sometimes disastrous consequences because impairing the normal balanced growth of the immature spine as well as the surrounding structures as the thorax and lung development with a restriction upon the pulmonary function. Subsequently the limitation of extensive spinal surgery on the growing spine appeared very clearly with time.

Knowing and practicing from the past when we had no instruments to be implanted to treat every kind of spinal deformities, but only pre-operative and post-operative cast to achieve the required immobilization, gave me the advantage to have some critical view about what it is done today in order to prepare the future. 


\section{The age of correction + fusion without instrumentation}

\section{Correction}

Any localized or more extended spinal deformities, no matter the etiologies, were corrected with more or less efficacy, thanks to various procedures including traction from head to lower limbs, sometimes directly on the bed, or thanks to specially designed frames like the Abbott or Risser ones, controlled with AP and lateral $\mathrm{X}$-rays.

\section{Stabilization}

Then the correction obtained was maintained and sometimes increased by a body cast with a sufficient collar to get an occipital/chin support to prevent collapsing, and a very good pelvic fitting with sometimes extension to one or both knees in order to control for example a significant correction of a pelvic obliquity. Sufficient windows were done in the front to allow the maximum of breathing possibilities, with a special attention to the pressure points around the brachial plexus levels on both sides. Of course, appropriate window in size was delineated on the back when the surgery was decided with this approach. Sometimes when the anterior surgery was necessary a large lateral window was done with special prepared points of connection with bolt and screws included in the cast in order to get an easy rebuilt of the cast in the immediate post op. Same principle was used when the approach was retro peritoneal for an Ant L5/S1 interbody fusion for example.

\section{Fusion}

At that time (still now in many places) the bone fusion is considered as the goal to be reached to get a sound stability of the correction. The fusion of the appropriate segments of the spine was determined according the pre-operative planning, generally from end vertebra to end vertebra with no hesitation to fuse to the sacrum when a significant correction of the pelvic obliquity was observed.

\section{Various techniques were used for posterior approach (1)}

The surgery was done inside the cast after a proper draping. The basis of that surgery was a decortication of the posterior elements according Hibbs, then Moe, Hall, Goldstein, including the facet joints fusion. An additional autogenous graft was done coming from the iliac crest. Albee in the mean-time was using an autogenous long tibial strut graft (harvested from the inner side of the tibial diaphysis (keeping intact the anterior tibial crest, to prevent a possible fracture), to get a more strong fixation with the great advantage to have a biological fusion at every level with the benefit of the rigidity of the cortical bone transplant until its reabsorption remodeling will be achieved. (I personally continue to use this principle in severe neuromuscular cases even when treated posteriorly with instrumentation). But for example, Cotrel and Cauchoix used at that time even for idiopathic cases a tibial autogenous graft and in other cases heterogeneous (from veal dis-specified) strut graft embedded and locked between the spinous processes of end vertebrae or the curve in order to reduce the post op immobilization.

\section{For the anterior approach techniques}

Personally, it is clear that the "small green book" written and published by Arthur Hodgson (2) was a great importance for me and probably many colleagues because he describes the easiest and safest way to approach the anterior aspect of the spine especially thanks to his great experience about the tuberculosis of the spine and Pott's pathology. With these principles in mind It is easy to approach from the front any place in the spine, with or without external traction or external fixation with or without cast. Especially the approach from the concave side for a kyphoscoliosis deformity demonstrate the perfect positioning of struts grafts (coming from tibia or fibula) even in a palisade fashion for kyphosis, because biomechanically they are exactly in the gravity alignment in front of the surgeon's eyes, when if you approach from the convex side you are far to see the good positioning and biomechanical proper alignment of the strut graft. Because at that time with no instrumentation a clear difference appeared between the anterior fusion obtained with the pure interbody fusion called in our slang language ("spread and butter") with no stability compared to the struts cortical embedded and locked between the vertebral plateau $\mathrm{X}$ very stable. In some more severe kyphosis where anterior decompression of the dural sac and spinal cord was necessary this technique of anterior struts in a palisade fashion very stable was another very reliable way to prevent complication thanks to its stability even if a complementary posterior fusion still without instrumentation was necessary to be done.

At the same time, during my residency some neuro surgeons like Gerald Guiot in Paris in front of the difficult case of a progressive paraplegia, secondary to a dystrophic kyphoscoliosis, develop an anterolateral approach of the 
spinal cord compression in order to obtain a real anterolateral cord transposition after resection of the antero lateral bulging of the vertebral bodies as well as the concave pedicles. At the end of the decompression, the dural sac and the spinal cord were lying outside of the canal on the concavity of the curve inside the thorax with one apical convex root hanging the cord with the dilemma to cut it to allow a better translation. Complete neurological recovery occurs after this surgery and a second stage of posterior fusion without instrumentation was done in situ without early complication, but after other 6 months of follow up a non-union appeared at the apex of this posterior fusion and you imagine what was my anxiety and difficulty when I had to approach again the front of the spine to realize the anterior fusion, the final result was ok with a solid front and back fusion. The lesson was that when doing such anterior decompression of the neural structures it is mandatory to do the anterior inter-bodies fusions at the same time.

\section{Post-operative immobilization}

For all these techniques without instrumentation, the postoperative immobilization to achieve the fusion is crucial and realized with generally the operative cast closed properly after the control of the wound healing. The duration of the cast recumbent was, according the length of the fused area, between 6 to 9 months. In some cases, standing was allowed after these 6 months. Then a new cast was realized in order to allow walking when it was possible and after 1 year a brace was used for another year.

\section{Results}

Even if the final result on the Cobb angle correction was not brilliant (less than $50 \%$ correction), we must recognize that the long term results of such treatments were particularly stable because of the quality of the bony fusion obtained thanks to generally a good balance given by the still a little bit malleable not too rigid bone mass when the patient after 6 months stand up and was submitted to gravity and subsequently compensate to achieve his personal alignment. These observations were among these that push me to describe later on the concept of the "Cône of Economy". But evidently the time necessary at that era to get the result was terribly long. It is why nowadays such strategy is exceptionally used but sometimes is able to give a solution to a particular problem.

\section{The era of spinal instrumentation}

If we remember the stammering from Hadra Silver Wires (year 1891) or the Chipault claws (year 1897), Lange (year 1910), Steel bars attached to vertebrae, with also the Wilson plates, ect., the real starting point of the spinal instrumentation surgery was done thanks to the revolutionary Harrington Instrumentation (year 1962) (3).

Initially designed by Paul Harrington as a correcting anti-collapsing device thanks to a notch ratchet's rod inserted between a Pedicle hook upward and a laminar hook downward located on the concave side at each end vertebra levels of the curve, and used without fusion like an "internal brace". Quickly, because the instability of the construct was observed Paul Harrington design the complete construct with concave distraction rod and convex compressive rod in association with the precise posterior fusion with additional autogenous graft already described, and subsequent cast immobilization for 6 months. Immediately adopted by most spine surgeons all over the world. Some refinements in its use were given by Pierre Stagnara and the "wake up test" in order to control the function of the spinal cord and its possible lesion thanks to the significant stretching power of the device, but also on a biomechanical point of view by Yves Cotrel transverse loading apical system (DTT) as soon as 1968, in order to decrease the length of post op cast immobilization.

In the meantime, Jacques Resina (year 1964), Alves Ferreira (year 1972) from Portugal and later Eduardo Luque (year 1973) from Mexico develop a posterior instrumentation with rods attached to the posterior arch of the vertebrae by sub laminar wires on each vertebra (SSI: segmental spine instrumentation) of the deformity planned to be fixed, with 2 major advantages reconstruction of a more physiological sagittal contour and no necessity of post-operative cast. It was also the time (1964/1977) of the fortuitous discovery by Raymond Roy Camille of the pedicular screw fixation (4) and correction of the deformity using posterior plates screwed on the pedicles.

On the other hand an anterior approach was developed by Alan Dwyer from Australia (5) as soon as 1968 compressing anteriorly the convexity of the curve after disc excision, modified by Zielke in 1973 (6) to improve the de-torsion of the scoliotic curve (Ventral Derotation spondylodese) and trying to reduce the kyphotic effect of the original anterior compression of the Dwyer technique. All these procedures were invented in order to give a better correction of the deformity as well as to drop down 
at maximum the post op care, each one having its own partisans and indications.

For posterior instrumentation, it is necessary to recognize at the end of the $70^{\text {th }}$ the work of Gordon Amstrong using 2 parallel Harrington rods (one concave one convex) in distraction to improve as the reduction as the stability.

Because at the end of the $70^{\text {th }}$ under the influence of René Perdriolle and the first computer reconstruction of a scoliotic spine that we got thanks to my common work with Henry Graf and Jerome Hecquet (Numerical engineer), the 3D aspect of the scoliotic deformity starts to be understood by the majority of the spine surgeons community. Because the continuous effort and genius of Yves Cotrel to find a stable and secure posterior metallic construct to avoid any post-operative immobilization lead to a proposal of a segmental construct. All was set to enter in the era of segmental instrumentation strategy. Because historical circumstances lead to join together our minds and work the so-called CD instrumentation and CD strategy appeared in 1983 (7). It was really an important turn, worldwide accepted, in the surgical treatment of the pediatric spine thanks to the segmental action obtained even at one level by the ability to act at one level separately from the next one (with proper use of selective location and orientation of hooks, and one year later of pedicle screws), thanks to the axial rotation of a pre-bent rod and all the strategies done according the analysis of the spine globally and segmentally. Of course, similar strategy was applied for anterior approach rod rotation and fusion. It is not excessive to say that CD instrumentation devices and strategies are still at the basis of all modern instrumentations for spinal deformities nowadays, and moreover the development of this instrumentation has opened widely the field of spinal surgery in adult hood as for elderly people thanks to the suppression of the post op cast. The goal of CD strategy and instrumentation was not to get the best Cobb angle because as said Alain Dimeglio: "the maximum of reduction is not always the optimum" and for me I explain that the most important part of a spine getting surgery for scoliosis is not the fused and instrumented one even if a significant curve remain in the bony block, but the parts left free of fusion above and below are the most important in order to achieve harmony and a dynamic $3 \mathrm{D}$ balance. So, for us the race for the minimum residual Cobb angle was not our goal.

For the neuro muscular pathology with pelvic obliquity we demonstrate very clearly that the best fixation to the pelvis was done with bilateral "Iliosacral" cannulated screw (8) from which the spinal construct is built allowing a very strong stable and reliable device with not any pull out observed in our personal experience over 200 cases, and a pseudarthrosis rate inferior to $2 \%$.

\section{The present aspect of spinal surgery in the pediatric age}

The present aspect of spinal surgery in the pediatric age is in relation with the development of the technology especially for the pedicle screws pushing some surgeons under the influence of Suk (9) from Seoul, to fit every vertebra of the curve with bilateral pedicle screws. This is for me ok for degenerative disorders or "de novo" scoliosis in the adults where the inductive disorder lies in the disco genic cascade, but not for pediatric developmental scoliosis secondary to neuro hormonal disorder with progressive structural deformities of the bony vertebrae structures themselves. The understanding of the basic deformity and the potential neurological risk of sub laminar metallic wires during insertion or extraction pushed Keyvan Mazda to introduce the so called "universal clamp" with a flexible plastic band slipped under the laminae of selected vertebrae to produce a real 2D translation on frontal and sagittal planes.

As well as for pediatric spine \& even very small young vertebrae especially designed laminar hooks and pedicle screws are built to fit perfectly the size and can be used for localized correction of a congenital malformation for example, or more extended use.

The major problem still now, is the introduction of a surgical treatment in case of failure of the not operative treatment for progressive scoliosis either from idiopathic, syndromic or neuro muscular etiology in the growing child. The reason of this difficult task is that we have to deal with keeping the most normal growth possible of the spinal structures as well as the growth of the lungs and thoracic cage. We know the disastrous seen when an extended sub-periosteal approach has been done at the thoracic or lumbar area, with subsequent sometimes severe restriction of the respiratory function; On the other hand, we know the iatrogenic deformities created with the crank shaft phenomenon very difficult to manage when it happen, and where the best treatment is prevention. It is why numerous attempts have been made from Harrington distraction rods without fusion, Luque trolley, Pediatric CD sub cutaneous trans muscular distraction rods with dominos, even for some of them, attachment on the ribs 
like VEPTER, others with spine fixation with apical pedicle screws like Shilla procedure. To prevent iterative surgeries and anesthesia's in order to re-tensioning of the distraction system, first, a unique expandable concave rod was designed and used without anesthesia or surgery at the out-patient clinic Then similar shape but magnetic device have been used with trans cutaneous lengthening systems, quickly evolved toward a dual rods magnetic powered device still in use. Some results were satisfying giving maintenance of the correction and partial result upon growth of the spinal structures. But it is well reported that the number of complications local or general was too high to be accepted as the recommended procedure.

It is why a new generation and philosophy appears taking in consideration all previous experiences done from the last 20 years: the Bipolar Mini invasive approach construct, under the strong influence of my pupil Lotfi Miladi (10).

\section{Technique}

After a preoperative preparation with halo traction or Elongation with a Stagnara cast the surgery is performed with per operative traction between head and lower limbs. Then at the levels chosen on the preoperative planning a strong upper fixation is realized bilaterally with 2 consecutives bilateral Supra laminar/Infra Pedicle claws on 2 consecutive vertebrae inserted with the minimum of soft tissues dissection and smooth penetration of the ligamentum flavum thanks to the blunt and delicate shape of the blades of the hooks. At the lower part either with 2 consecutive pedicle screws in case of idiopathic cases or bilateral mini invasive "ilio-sacral screws" for neuromuscular spine with pelvic obliquity. A bilateral properly bent rod is inserted trans muscular between the 2 poles using generally a domino on each side to facilitate fitting of the rod., The strategy is to insert the rods to remain away from the apex of the deformity and to get elongation slowly, progressively thanks to the "Click Dominos" devices, allowing elongation with movements of the patient, spontaneous growth of the vertebrae, or if necessary some traction done on the lower limbs without any anesthesia at the clinic. So, no fusion is done any time. The stiffness and strength of the construct is done by these 2 parallel rods or even 4 if necessary, always fitted bipolar trans muscular and also by the characteristics of the metal Titanium, Stainless steel or Cobalt Chrome.

The philosophy is to create a strong permanent anticollapsing device, sufficient to avoid any external protection by brace or cast, and able to maintain the spine and patient until maturity, to get a kind of spontaneous fusion of the spinal elements or sufficient stiffness to avoid a secondary more or less late surgical fusion.

\section{Results}

The oldest case done on a now 22-year-old patient where initial surgery was done at age 9 years old has shown such evolution with proof of such fusions at the facet joints levels or bone formation along the rods with a pleasant maintenance of the cosmetic correction of the deformity. For the neuro muscular and syndromic etiologies (more than 100 cases with sufficient 5 years follow up, the immediate result thanks to this immediate anti-collapsing technique and immediate stability without external support is quite spectacular for the sitting function. We observe quickly the changes on the general status, breathing, weight, urinary and bowel functions.

In addition, it is amazing to control the progressive reduction of the pelvic obliquity for example on the $\mathrm{CP}$ patients. In addition, the serious probability that no other extensive surgery for fusion will be necessary when reached the maturity is another factor extremely interesting as for the patient quality of life as for the cost as for the society. Of course, in case of failure or not sufficient result the classic fusion and adequate instrumentation will be always available.

\section{Let us look now toward the future}

\section{The indications for surgery for pediatric spinal deformities will probably drop down for many reasons}

Prevention campaign before conception for example supplementation in folates to prevent anomalies of neural tube, or limitations in alcohol, drug or tobacco consumption. Moreover, it appears that many congenital spinal malformations can be secondary to a fortuitous episode of a more or less pronounced stress occurred in the first 3 week after conception, as recognized during the not guilty delicate enquiries of the first contact patientphysician. This is reproducible experimentally in the mouse, conducting to similar malformations for similar stress in time and intensity.

Prenatal detection of congenital anomalies thanks to legal echographia's during pregnancy, with the ethical problems that are coming from, to propose to the parents the interruption.

Prevention of severe or mild prematurity in order to drop 
the number of cerebral palsy sequels, often consequences of the change in the society habits such as voluntary delay in the pregnancy for personal wishes with often necessity to use repeated FIV to become pregnant for the mother, forgetting that the physiological age for pregnancy is between 18 and 35 years old. We hope that a national campaign on this topic will decrease such incidence.

Early detection of the progressive forms of Idiopathic scoliosis thanks to the severity index measurement done at the first Bi planar EOS imaging system done. This allow to treat the patient with a mild deformity (sometimes less than $15^{\circ} \mathrm{Cobb}$ ) only at night for example, by a correcting cast or brace looking more on the de-torsion than to the Cobb Angle correction. Subsequently when used widely in the country the number of cases ending with spinal surgery will decrease significantly.

\section{The surgical correction of localized established congenital anomalies will remain in the growing age}

For example, hemi-vertebra resection or front and back fusion for dislocated congenital spine with or without instrumentation, or with or without neural structures decompression. The improvement of the technology helps nicely to obtain better reduction, but in most of the cases post-operative cast remain mandatory because very easy to use by the nursing staff or the parents giving security for the medical point of view. The consequence is that teaching of a proper and safe way to do a cast in a young child must be transmitted by the senior surgeons, because serial casting properly done remain the first weapon against a progressive scoliosis in the young child.

\section{The surgical correction on extended deformities of any etiologies touching the entire spine with or without pelvic obliquity, in case of failure of a not operative treatment}

For such deformities up to 6/7 years old the not operative treatment is still up to date with corrective cast or/ and braces generally done with anesthesia. When the development of the spinal and thoracic structures seems sufficient, it is the exact indication of the Bipolar technique already escribed with its present and future advantages.

\section{For spinal deformities established and seen later at the pre or adolescent age}

It is clear that the anterior approach is more and more forsaken for the posterior one, because the extension of the approach laterally sometimes on both side at one or more levels allow to get a good exposure of the front of the spine. Nevertheless, in our practice, the anterior approach seems useful and safer and more sound biomechanically speaking, every time we have to realize a support on the anterior vertebral bodies, especially from the concave side approach for kyphoscoliosis or direct lateral approach for pure kyphosis and necessity to perform anterior decompression of the neural elements or in some dystrophic spines as NF1, or finally when a circumferential vision is necessary for some extensive tumors.

For the most frequent pathology as idiopathic scoliosis when a surgical indication is proposed the posterior approach is chosen with today in the mind of most of the pediatric spine surgeons, the goal to reduce at maximum the Cobb angle while restoring the best possible sagittal alignment. It is my personal hope for the future that it will turn toward the research for harmony in the 3 planes especially looking on the importance of the horizontal plane correction. The horizontal plane not understood as the one given by one CT scan slice but by the $3 \mathrm{D}$ piling up vision of the successive vertebrae with their surrounding masses aligned on the gravity line of the entire body. The use of the $3 \mathrm{D}$ vertebral vectors concept and its measurements as proposed by Tamas Illes will help a lot to visualize, analyze, and quantify the deformity and its correction with a much better valuation than the co called "Gold Standard Cobb angle" that measure in reality only the collapsing factor of the spine. In addition, these $3 \mathrm{D}$ vectors help also to determine the levels to be fused thanks to the flexibility checked at the levels of the disc spaces.

\section{About the strategy for instrumentation}

The use of pedicles screws bilaterally at every level will progressively decline, as already done, in regard of the complications observed such as: extension of the instrumented area secondary to excessive correction of the deformity and subsequent PJK or DJK. The evolution going to less correction and use of hybrid construct with or without use of the Universal clamps or use of more strategic located hooks is renewing.

In case of very severe deformities, the use of vertebral column resection (VCR) will remain exceptional if not abandoned for idiopathic cases because of the high risk of complications, and because prolonged halo traction and multiples posterior facets joints release, with very seldom 
anterior discectomies have demonstrated their efficacy with a clear limitation of the post op complications thanks the good pre-operative preparation and the limited goals for reductions looking mainly on the general and respiratory status improvement.

On the same concept to assess the result of the correction of a pelvic obliquity, instead of measuring angles, it is more sound and close to the practical goal of the surgery, to evaluate the pressure forces under the buttocks and the tights of the patient, as in a static as in a dynamic fashion as I proposed experimentally in 1975 , but easy to achieve today thanks to the modern technology.

On the same topic, I continue to not understand why in case of lumbosacral fixation, the" Ilio-Sacral screw", that I consider with all of my pupils the most reliable, safe and best instrumentation to control the "Pelvic Vertebra", is not accepted by the orthopedic population. Probably it was because the direction of the guide wire for the cannulated screw was difficult to imagine in the mind of many surgeons to escape the neural canal and the L5 nerve root, but now with the navigation tools, this fear will disappear. In reality this technique was not well described in details in the publications and many surgeons imagine that this screw transfixes the Sacro-iliac joint? In reality it is a real transversal sacral screw with all the advantages of such location, but inserted through an iliac wing hole clearly posterior of the SI joint.

\section{For trauma in childhood or adolescent age}

The nice classifications established are much better understood and used as a real "check list", especially when a surgical treatment is indicated and then the use of the pedicle screws (with all its adaptation according the age of the patient) is a great improvement to achieve stability even if a decompression of the dura and spinal cord had been necessary. Nevertheless, frequently in childhood a postoperative cast is a wise precaution for 6 to 8 weeks.

\section{For high grade spondylolisthesis}

The discussion remains between those doing an in-situ fusion and those who are in favor of some kind of reduction. Between those who want to reduce the deformity, the discussion remains between those doing an open reduction with wide laminectomy, release of both L 5 roots, resection of the sacral dome, reduction with an L5/S1 interbody fusion with graft or cage and pedicles instrumentation with postero-lateral fusion. All this is done under the control of a precise neuro and roots monitoring.

The other proposal is to perform and awake progressive reduction thanks to halo cranial traction and suspension of the pelvis with a large strap under the buttocks lifting progressively the pelvis in order to reduce the lumbo-sacral kyphosis. We check the correction with a lateral X-ray control and of course we have a permanent clinical control of the possible neurological or roots involvements and we observed almost always a recovery when they were present at the first exam. When, in some hours or days the reduction of the lumbo-sacral kyphosis is obtained completely (LS angle $>110^{\circ}$ ) or partially (LS angle $>110^{\circ}>90^{\circ}$ ) (we don't look about the $\%$ of slippage) we perform a body cast including both thighs. If the LS angle is $>$ or $=110^{\circ}$ a posterior window is done allowing to do a posterior fusion L4/Sacrum through a Wiltse approach without opening the canal, with autogenous graft from iliac crest. When the LS angle is $<$ or $=90^{\circ}$ the cast is cut longitudinally. The patient lies in the posterior half of the cast (so in reduced position). Then after a Pfannenstiel retroperitoneal approach, 3 anterior retroperitoneal strut graft L5/S2 tibial graft are inserted after L5/S1 disc excision. Then the anterior part of the cast is repositioned and cast closed. Simultaneously the postero-lateral fusion is done according the same strategy as previously presented. Neural and roots monitoring is of course can be used during surgery. The post op cast in both situations is kept recumbent for 3 months then a short body cast or body brace allow walking for other 3 months. It is clear that the amount of complications is significantly less in this second not aggressive neither instrumented technique, and the clinical and functional results confirmed excellent with 20-year follow-up.

\section{Conclusions}

Despite the strong development of new technologies about the instrumentation, simulation, and navigation system to make the surgical act safer and safer whatever the localization at the spine level, some basic recommendations remain to be recall again.

Spine is an organ and must be understood from head to pelvis suspended over 2 femoral heads and lower limbs, working like a reverse pendulum working $3 \mathrm{D}$ all the time according the Cone of economy concept, demonstrating the importance of dynamic versus static evaluations.

Any surgery at any level of the spine produce in a growing spine a more or less extended fusion, with 
consequences on the growing structures: the younger the age of the lesion the worst are the consequences. This impair the development of the adjacent elements often vital such as lungs and respiratory function, leading from time to time to disasters. Subsequently the pediatric orthopedic surgeon must remember that any time he decides a surgical indication on a growing spine.

The most important part of a spine undergoing a spinal instrumentation is not the instrumented one but the one not instrumented left free above and below especially because these parts works to give compensation, movements and balance in $3 \mathrm{D}$ to give permanent adaptation. This advice forgotten can lead to difficult situations to be solved later on.

Despite the major improvement in surgical techniques thanks to the development and expertise in pediatric anesthesia and spinal cord monitoring allowing to address more and more difficult cases, the most important message for the pediatric orthopedic surgeon dealing with a deformity of the spine is to reach the goal of Harmony as well for the external 3D aspec of the patient as well as for the dynamic balance in the $3 \mathrm{D}$ from head to feet, because it is the best guarantee for the future functions in the adulthood and elderly time of the life.

\section{Acknowledgments}

None.

\section{Footnote}

Conflicts of Interest: The author has no conflicts of interest to declare.

Etbical Statement: The author is accountable for all aspects of the work in ensuring that questions related to the accuracy or integrity of any part of the work are appropriately investigated and resolved.

\section{References}

1. Hall JE. Spine surgery before and after Paul Harrington. Spine (Phila Pa 1976) 1998;23:1356-61.

2. Hodgson AR, Stock FE. Anterior spinal fusion a preliminary communication on the radical treatment of Pott's disease and Pott's paraplegia. Br J Surg 1956;44:266-75.

3. Harrington PR. Treatment of scoliosis. Correction and internal fixation by spine instrumentation. J Bone Joint Surg Am 1962;44-A:591-610.

4. Roy-Camille R, Roy-Camille M, Demeulenaere C. Osteosynthesis of dorsal, lumbar, and lumbosacral spine with metallic plates screwed into vertebral pedicles and articular apophyses. Presse Med 1970;78:1447-8.

5. Dwyer AF, Newton NC, Sherwood AA. An anterior approach to scoliosis. A preliminary report. Clin Orthop Relat Res 1969;62:192-202.

6. Zielke $\mathrm{K}$, Berthet A. VDS--ventral derotation spondylodesis--preliminary report on 58 cases. Beitr Orthop Traumatol 1978;25:85-103.

7. Cotrel Y, Dubousset J. A new technic for segmental spinal osteosynthesis using the posterior approach. Rev Chir Orthop Reparatrice Appar Mot 1984;70:489-94.

8. Miladi LT, Ghanem IB, Draoui MM, et al. Iliosacral screw fixation for pelvic obliquity in neuromuscular scoliosis. A long-term follow-up study. Spine (Phila Pa 1976) 1997;22:1722-9.

9. Suk SI, Lee CK, Kim WJ, et al. Segmental pedicle screw fixation in the treatment of thoracic idiopathic scoliosis. Spine (Phila Pa 1976) 1995;20:1399-405.

10. Miladi L, Gaume M, Khouri N, et al. Minimally Invasive Surgery for Neuromuscular Scoliosis: Results and Complications in a Series of One Hundred Patients. Spine (Phila Pa 1976) 2018;43:E968-75.
Cite this article as: Dubousset J. Past, present, and future in pediatric spinal surgery. Ann Transl Med 2020;8(2):36. doi: 10.21037/atm.2019.08.13 